\title{
A multiplex real-time PCR assay for routine diagnosis of bacterial vaginosis
}

\author{
J. G. Kusters ${ }^{1}$ - E. A. Reuland ${ }^{2}$ - S. Bouter ${ }^{3}$ - P. Koenig ${ }^{3}$. \\ J. W. Dorigo-Zetsma ${ }^{3}$
}

Received: 6 March 2015 / Accepted: 13 May 2015 /Published online: 5 July 2015

(C) The Author(s) 2015. This article is published with open access at Springerlink.com

\begin{abstract}
A semi-quantitative multiplex PCR assay for the diagnosis of bacterial vaginosis (BV) was evaluated in a prospective study in a population of Dutch women with complaints of abnormal vaginal discharge. The PCR targets Gardnerella vaginalis, Atopobium vaginae, Megasphaera phylotype 1, Lactobacillus crispatus and Lactobacillus iners. Together with a short questionnaire, a vaginal swab for PCR and vaginal smear for microscopy were taken by their general practitioner or gynaecologist. Data from 151 women (median age 32) were available. Nugent Score (NS) was used to classify the samples and 83 samples were classified as normal (NS $0-3), 13$ as intermediate (NS 4-6), and 55 as bacterial vaginosis (NS 7-10). In women with a NS of 7-10, PCR detected Gardnerella vaginalis, Atopobium vaginae and Megasphaera phylotype 1 in respectively, $96 \%, 87 \%$ and $60 \%$, whereas in women with a NS of 1-3 these species were detected in $27 \%$, $6 \%$ and $2 \%(P<0.001)$. A ratio of Lactobacillus crispatus over Lactobacillus iners of $<1$ (as calculated from the quantification cycle value (Cq)) was present in women with a NS of $7-10$ in $66 \%$ versus $33 \%$ in women with a NS of $1-3(P$ $<0.001)$. The BV-PCR displayed a sensitivity of $92 \%$ and specificity of $96 \%$ with a positive predictive value of $94 \%$ and a negative predictive value of $95 \%$. The Lactobacillus-
\end{abstract}

J. G. Kusters

h.kusters@umcutrecht.nl

1 Department of Medical Microbiology, University Medical Center Utrecht, G.04.614, P.O. Box 85500, 3508

GA Utrecht, The Netherlands

2 Medical Microbiology \& Infection Control, VU University Medical Center, Amsterdam, Netherlands

3 Department of Medical Microbiology, Tergooi Hospital, Hilversum, Netherlands index improved the correct classification of samples where only one of the other bacterial species was detected. Compared to the Nugent Score this multiplex qPCR offers a convenient tool for performing observer independent diagnosis of BV.

\section{Introduction}

Abnormal vaginal discharge is the most common gynaecological condition for which a general practitioner is consulted. In the Netherlands there is an estimated incidence of 40-50 per 1,000 female patients per annum [1]. Trichomonas infections, Candida albicans infections, and bacterial vaginosis (BV) are among the top three causes of abnormal vaginal discharge. Moreover, BV has been associated with increased risk of adverse sequelae, such as preterm delivery, low birth weight, pelvic inflammatory disease and enhanced risk of acquiring STI [2].

Classically, BV is diagnosed according to the full criteria of Amsel et al. [3] or solely based on microscopy using either direct (wet mount) microscopy or microscopic examination of a Gram-stained vaginal specimen [4]. Routine culture of genital specimen reveals only part of the vaginal microbiota, and the presence of a culturable, well-known BV-associated species like Gardnerella vaginalis is relatively unspecific for diagnosis of BV [5].

Recently, cultivation-independent methods like PCR have revealed that the presence of newly discovered vaginal species such as Atopobium vaginae, unknown Clostridiales and Megasphaera spp., might be diagnostic of BV [6]. As the different vaginal species can be part of the normal vaginal microbiota, quantification can greatly improve the performance of these PCR-assays for diagnosis of BV [7-10]. Furthermore, the microscopic 
examination of vaginal specimen by the Nugent method is based on the relative abundance of Lactobacillus morphotypes (large Gram positive rods) compared to the abundance of bacterial morphotypes suggestive of BV, but it does not discriminate between the different Lactobacillus species. However, the ratio in which different Lactobacillus species appear in vaginal specimen can be predictive for normal or abnormal microflora: L. crispatus is found mainly in females with healthy vaginal microbiota, whereas $L$. iners is detected both in healthy and disturbed vaginal microbiota $[9,11-14]$. The present study describes the development of a multiplex qPCR assay for the semi-quantitative detection of well-recognized BV-associated marker organisms and different Lactobacillus species and its evaluation in a clinical setting.

\section{Materials and methods}

\section{Study population}

The study was approved by the local ethical board of the investigating institute, Tergooi Hospital, Hilversum, the Netherlands, and written informed consent was obtained from participants.

A total of 159 women with self-reported complaints of abnormal vaginal discharge presenting to their general practitioner or gynaecologist between April 2009 and April 2010 were enrolled in the study. All enrolled were $\geq 18$ years of age and had not received antibiotics or vaginal medications for at least 14 days prior to enrolment. The median age was 32 (range 18-62 years) and $>95 \%$ were Dutch Caucasians. At the time of enrolment all participants completed a short questionnaire, and both a vaginal swab for PCR (Female multiCollect Specimen Collection Kit; Abbott Molecular Inc., Des Plaines, IL, USA) and vaginal smear for microscopy were taken by the physician. Only if requested by the physician additional routine microbiological testing was performed. Evaluation could not be completed for eight of the patients, resulting in 151 patients for evaluation.

\section{PCR set-up}

Based on the literature and preliminary studies five bacterial species were selected for a multiplex real time PCR setup. These included three bacterial species (Atopobium vaginae, Gardnerella vaginalis, and Megasphaera phylotype 1) associated with bacterial vaginosis and two Lactobacillus species (L. crispatus and L. iners) detected both in the normal as well as in the disturbed vaginal microbiota $[5-7,11]$.
DNA was isolated from the swabs by automated DNA extraction on an Abbott M2000sp (Abbott, Illinois, USA) using the standard Chlamydia trachomatis/Neisseria gonorrhoeae DNA extraction protocol as advised by the manufacturer. The presence of the above-mentioned five bacterial species was established by an in-house semi quantitative real-time PCR. The specificity of the primer sets and probes (Table 1) was checked with PRIMER BLAST (http://www.ncbi.nlm.nih.gov/tools/primer-blast/) and all sets used were found to be highly specific for their intended targets. In addition we tested our multiplex qPCR with a large collection of strains both for each of the bacterial targets as well as with related bacterial species, and only the intended targets would show up as positive (data not shown). The qPCR reactions (see Table 1 for details) were performed as two multiplex reactions in a Corbett RotorGene 6000 PCR machine (Qiagen, Hilden, Germany) using Qiagen QuantiTect Multiplex Taq polymerase and corresponding buffers as advised by the manufacturer. Primer concentration was $0.5 \mu \mathrm{M}$ for all forward and reverse primers and $0.25 \mu \mathrm{M}$ for all probes. Reactions were performed in a final volume of $20 \mu 1$ containing $5 \mu \mathrm{l}$ DNA eluate. PCR conditions consisted of a heat-activation of the Taq polymerase of $95^{\circ} \mathrm{C}$ for $10 \mathrm{mi}$ nutes followed by 40 cycles of amplification. Each amplification cycle consisted of a denaturation step at $95^{\circ} \mathrm{C}$ for 15 seconds and an annealing/amplification step at $55{ }^{\circ} \mathrm{C}$ for one minute. In all qPCR runs positive controls for all targets as well as negative controls $\left(\mathrm{H}_{2} \mathrm{O}\right.$ instead of purified sample DNA) were always included. In addition multiplex I (See Table 1) included a primer/probe set for detection of human $\beta$-globulin DNA to check for adequate sample collection and preservation and isolation of DNA of sufficient quality. The qPCR results of clinical samples were only considered valid if the $\beta$-globulin PCR resulted in a quantification cycle $(\mathrm{Cq})$ value $\leq 35$ and second derivatives of the amplification plots were calculated to determine the fractional qPCR cycle value. Bacterial PCRs were scored as positive if a clear S-curve was observed in the respective amplification plot.

\section{Lactobacillus index}

For the presence of Lactobacillus species an index was calculated: in case of $\geq 5 \mathrm{Cq}$ value difference between the two Lactobacillus species and a higher load of L. iners detected, the 'Lactobacillus-index' (L-index) is $<1$ (indicating a shift to disturbed vaginal microbiota); in case a higher load of $L$. crispatus was detected, the L-index is $>1$ (indicating healthy vaginal microbiota); in case both Lactobacillus species were detected in the same amounts, or both were absent, the L-index $=1$ (undetermined vaginal microbiota). 
Table 1 Primer sets and amplicon length of the two multiplex PCRs

\begin{tabular}{|c|c|c|c|}
\hline PCR target & Primer name & Oligo composition $\left(5^{\prime}-3^{\prime}\right)^{\mathrm{a}}$ & Amplicon size \\
\hline \multicolumn{4}{|l|}{ Multiplex 1} \\
\hline \multirow[t]{2}{*}{$\beta$-globulin ${ }^{\mathrm{b}}$} & $\begin{array}{l}\text { Forward } \\
\text { Probe }\end{array}$ & $\begin{array}{l}\text { GAAGAGCCAAGGACAGGTAC } \\
\text { [Cy5]TCTGCCGTTACTGCCCTGT }\end{array}$ & \multirow[t]{2}{*}{$268 \mathrm{bp}$} \\
\hline & Reverse & CAACTTCATCCACGTTCACC & \\
\hline \multirow[t]{2}{*}{ L. iners } & $\begin{array}{l}\text { Forward } \\
\text { Probe }\end{array}$ & $\begin{array}{l}\text { AGTCTGCCTTGAAGATCGG } \\
\text { [FAM]CCAAGAGATCGGGATAACAC } \\
\text { CT }\end{array}$ & \multirow[t]{2}{*}{$166 \mathrm{bp}$} \\
\hline & Reverse & CTTTTAAACAGTTGATAGGCATCATC & \\
\hline \multirow[t]{2}{*}{ L. crispatus } & $\begin{array}{l}\text { Forward } \\
\text { Probe }\end{array}$ & $\begin{array}{l}\text { AACTAACAGATTTACTTCGGTAATGA } \\
\text { [ROX]CCCATAGTCTGGGATACCACTT }\end{array}$ & \multirow[t]{2}{*}{$145 \mathrm{bp}$} \\
\hline & Reverse & AGCTGATCATGCGATCTGC & \\
\hline \multicolumn{4}{|l|}{ Multiplex 2} \\
\hline \multirow[t]{2}{*}{ A. vaginae } & $\begin{array}{l}\text { Forward } \\
\text { Probe }\end{array}$ & $\begin{array}{l}\text { TAGGTCAGGAGTTAAATCTG } \\
{[\text { HEX]CTACCAGACTCAAGCCTGCC }}\end{array}$ & \multirow[t]{2}{*}{$155 \mathrm{bp}$} \\
\hline & Reverse & TCATGGCCCAGAAGACCGCC & \\
\hline \multirow[t]{2}{*}{ G. vaginalis } & $\begin{array}{l}\text { Forward } \\
\text { Probe }\end{array}$ & $\begin{array}{l}\text { GCGGGCTAGAGTGCA } \\
{[\text { ROX]CTTCTCAGCGTCAGTAACAGC }}\end{array}$ & \multirow[t]{2}{*}{$206 \mathrm{bp}$} \\
\hline & Reverse & ACCCGTGGAATGGGCC & \\
\hline \multirow[t]{2}{*}{ Megasphaera phylotype 1} & $\begin{array}{l}\text { Forward } \\
\text { Probe }\end{array}$ & $\begin{array}{l}\text { GATGCCAACAGTATCCGTCCG } \\
\text { [FAM]ACAGACTTACCGAACCGCCT }\end{array}$ & \multirow[t]{2}{*}{$208 \mathrm{bp}$} \\
\hline & Reverse & СCTCTCCGACACTCAAGTTCGA & \\
\hline
\end{tabular}

${ }^{a}$ Primers and probes were obtained from TIB MOLBIOL GmbH, Berlin, Germany. Cyanine 5 (Cy5), Fluorescein (FAM), X-Rhodamin (ROX) and Hexachlorfluorescein (HEX) were used as the $5^{\prime}$-coupled reporter fluorophores of the hydrolysis probes used in the multiplex PCR reaction, and the 3'-coupled Black Hole Quencers (BHQ1 and BHQ2) as quenchers

${ }^{\mathrm{b}} \beta$-globulin PCR was used as a sample and DNA/PCR quality control. See the "Materials and methods" section for explanation

\section{Vaginal smears}

Vaginal smears were Gram-stained and scored using Nugent Score (NS). Based on their NS patients were subdivided in three groups: normal vaginal microbiota (NS 0-3), intermediate vaginal microbiota (NS 4-6) and bacterial vaginosis (NS 7-10). Slides were scored independently by two experienced technicians. In case of discordant results $(n=43 / 151 ; 28 \%)$, slides were re-examined by the same technicians, unaware of their previous score. For vaginal smears remaining with discordant results after this second round $(n=8)$, the microscopists sat together and came to consensus after a final round of revisions.

\section{Statistical analysis}

The Cq values for both Lactobacillus species were determined and when appropriate the L-index (ratio of L. crispatus over L. iners) was calculated from these $\mathrm{Cq}$ values as described above. Statistical analyses were performed with SPSS, version 20.0. Proportions were compared using Pearson's $X^{2}$, Fisher's exact test and Mann-Whitney U test where appropriate. Scatter plots were prepared using Prism V6.0 for Windows (GraphPad Software Inc., San Diego, CA).

\section{Results}

\section{Characteristics of women enrolled in the study}

For 151 women, a vaginal swab for PCR, a vaginal smear for microscopy, informed consent and a completed questionnaire were received. For 149 of them, additional microbiological testing, i.e. routine culture of the genital specimen $(n=149)$ and molecular diagnostics for Chlamydia trachomatis and Neisseria gonorrhoeae $(n=114)$, Trichomonas vaginalis or herpes simplex virus (HSV), was performed.

Of the 151 women, 83 (55\%) had normal vaginal microbiota, $13(9 \%)$ intermediate vaginal microbiota and $55(36 \%)$ had bacterial vaginosis according to NS. The clinical and laboratory characteristics of this population are shown in Table 2. Sexually transmitted infections (STIs) among these 151 patients were uncommon; one woman had $C$. trachomatis, one had $N$. gonorrhoeae, three had T. vaginalis and three had HSV infection. Further pathogens detected were: Candida species, mostly C. albicans (in 39 women), Haemophilus influenzae (in three), and Group A $\beta$-hemolytic Streptococcus (in two). Seventy-four women used (oral) contraceptives (OC), eight were pregnant and 115 had visited their physician for abnormal vaginal discharge in the preceding two years. 
Table 2 Clinical and laboratory findings in 151 females with abnormal vaginal discharge

\begin{tabular}{|c|c|c|c|c|}
\hline Patient sample characteristics & $\begin{array}{l}\text { Nugent score } 0-3 \\
(n=83)\end{array}$ & $\begin{array}{l}\text { Nugent score 4-6 } \\
(n=13)\end{array}$ & $\begin{array}{l}\text { Nugent score } 7-10 \\
(n=55)\end{array}$ & P-value \\
\hline Age (median) when obtaining sample ${ }^{a}$ & $31.7(25-40.4)$ & $32.3(22.4-41.5)$ & $33.1(24.8-41.3)$ & $0.981^{\mathrm{c}}$ \\
\hline Patients using contraceptives ${ }^{\mathrm{b}}$ (data based on $149 / 151$ patients) & $\begin{array}{l}39(50 \%) \\
35 \text { OC, } \\
4 \text { IUD/other }\end{array}$ & $\begin{array}{l}6(46 \%) \\
5 \text { OC, } \\
1 \text { IUD/other }\end{array}$ & $\begin{array}{l}29(58 \%) \\
19 \text { OC, } \\
10 \text { IUD/other }\end{array}$ & $\begin{array}{l}0.376 \\
0.442 \\
0.009\end{array}$ \\
\hline \multicolumn{5}{|l|}{ Vaginal discharge } \\
\hline $\begin{array}{l}\text { Patients }{ }^{\mathrm{b}} \text { with known prior events of abnormal vaginal discharge } \\
\text { (data based on } 146 / 151 \mathrm{pts} \text { ) } \\
\text { Known non-BV cause of vaginal discharge }\end{array}$ & $50(63 \%)$ & $11(85 \%)$ & $32(60 \%)$ & 0.805 \\
\hline -Candida sp. & $21(25 \%)$ & $5(39 \%)$ & $13(24 \%)$ & 0.855 \\
\hline -Other & $7(8 \%)$ & $1(8 \%)$ & $2(4 \%)$ & 0.483 \\
\hline \multicolumn{5}{|l|}{ Presence of Gardnerella/Atopobium/Megasphaera } \\
\hline Gardnerella (total no. of positive samples) & $22(27 \%)$ & $8(62 \%)$ & $53(96 \%)$ & $<0.001$ \\
\hline No. of samples with $\mathrm{Cq} \leq 20$ & $6(7 \%)$ & $3(23 \%)$ & $30(55 \%)$ & $<0.001$ \\
\hline Atopobium (total no. of positive samples) & $5(6 \%)$ & $4(31 \%)$ & $48(87 \%)$ & $<0.001$ \\
\hline No. of samples with $\mathrm{Cq} \leq 25$ & $1(1 \%)$ & $3(23 \%)$ & $29(53 \%)$ & $<0.001$ \\
\hline Megasphaera (total no. of positive samples) & $2(2 \%)$ & $2(15 \%)$ & $33(60 \%)$ & $<0.001$ \\
\hline No. of samples with $\mathrm{Cq} \leq 25$ & 0 & $1(8 \%)$ & $21(38 \%)$ & $<0.001$ \\
\hline Lactobacillus index $<1$ & $27(33 \%)$ & $6(46 \%)$ & $36(66 \%)$ & $<0.001$ \\
\hline
\end{tabular}

$O C$ oral contraceptives, IUD intra uterine device

$P$-values are a comparison between women with NS 0-3 and those with NS 7-10

${ }^{\text {a }}$ Variables are denoted as median (interquartile range)

${ }^{\mathrm{b}}$ Data are only available for the indicated subset of patient samples

${ }^{\mathrm{c}}$ Group differences were tested with the Mann-Whitney U test

\section{PCR-based detection of $G$. vaginalis, $A$. vaginae, Megasphaera, L. crispatus and L. iners}

In women with BV (NS 7-10), G. vaginalis, A. vaginae, and Megasphaera phylotype 1 were present in $96 \%, 87 \%$ and $60 \%$ of the vaginal specimens, respectively, whereas in women with normal vaginal microbiota (NS 0-3) these bacteria were detected in, respectively, $27 \%, 6 \%$ and $2 \%(P$ $<0.001)$. To establish the quantitative relationship between bacterial load and NS, bacterial loads were plotted against the NS in scatter plots and the median $\mathrm{Cq}$ value at which the bacterium was first detected among all samples was calculated (Fig. 1). Median Cq values for G. vaginalis, A. vaginae, and Megasphaera phylotype 1 were 20, 25 and 25, respectively. Higher loads of $G$. vaginalis $(\mathrm{Cq} \leq 20)$, of $A$. vaginae $(\mathrm{Cq} \leq 25)$, and of Megasphaera $(\mathrm{Cq} \leq 25)$ were significantly more common in women with BV than in women with normal vaginal microbiota $(P<0.001)$ (Table 2$)$. An L-index $<1$ (shift to disturbed vaginal microbiota) was present in women with $\mathrm{BV}$ in $66 \%$, compared to $33 \%$ in women with normal vaginal microbiota according to NS $(0-3)(P<0.001)$.

The proportion of women with $G$. vaginalis, $A$. vaginae, Megasphaera phylotype1 detected by PCR and the calculated L-index, stratified according to NS, are shown in Table 2.

\section{BV-PCR assay validation}

Subsequently, the multiplex qPCR assay for molecular diagnosis of BV (BV-PCR) was compared with the NS as reference method for the diagnosis of BV. BV-PCR was scored positive in case $\geq 2 \mathrm{BV}$-associated bacteria were detected, regardless of the L-index; BV-PCR was scored negative in case no BV-associated bacterium was detected, regardless of the L-index or one BV-associated bacterium was detected and L-index $\geq 1$; BV-PCR was scored indeterminate in case one $\mathrm{BV}$-associated bacterium was detected and L-index $<1$. Thus 55 samples were scored BV-PCR positive, $86 \mathrm{BV}-\mathrm{PCR}$ negative and ten BV-PCR indeterminate. A correlation between BV-PCR scores (either positive, indeterminate, or negative) and the corresponding Nugent scores (bacterial vaginosis, intermediate, or normal) of the samples was observed with negative PCR samples almost always having a normal NS and vice versa (not shown). Sensitivity and specificity, PPV and NPV of the BV-PCR was calculated using all samples $(n=131)$ for which both NS and BV-PCR results were conclusive (either positive or negative) for diagnosis of BV. The BV-PCR was highly sensitive: $92 \%$ and specific $96 \%$ for diagnosis of BV, with a PPV of $94 \%$ and NPV of $95 \%$ for predicting $\mathrm{BV}$. 
G. vaginalis

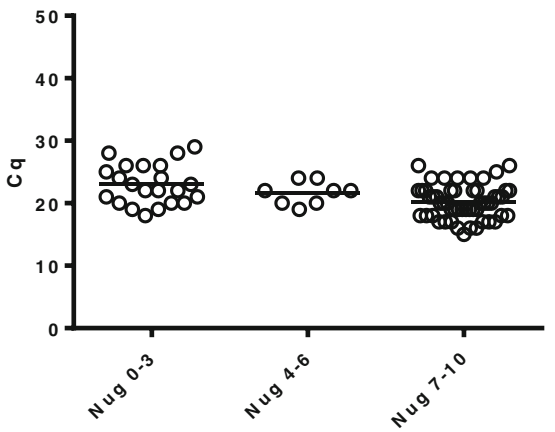

L. crispatus

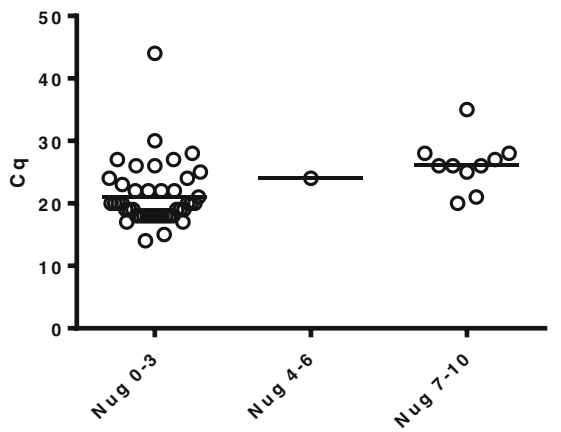

Megasphaera phylotype I

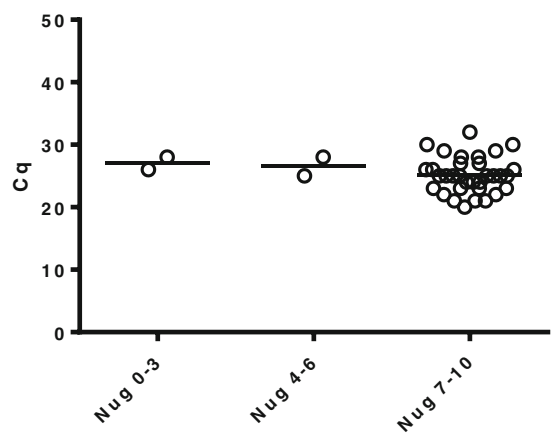

L. iners

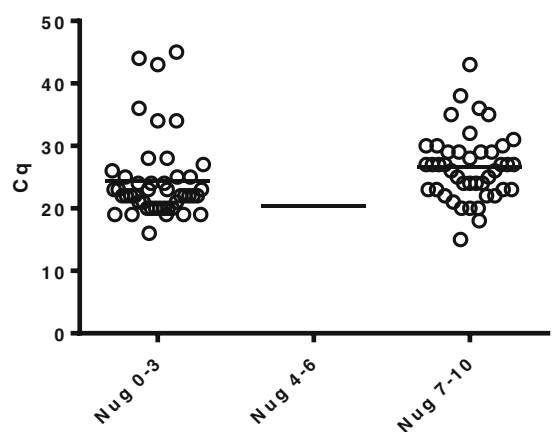

A. vaginae

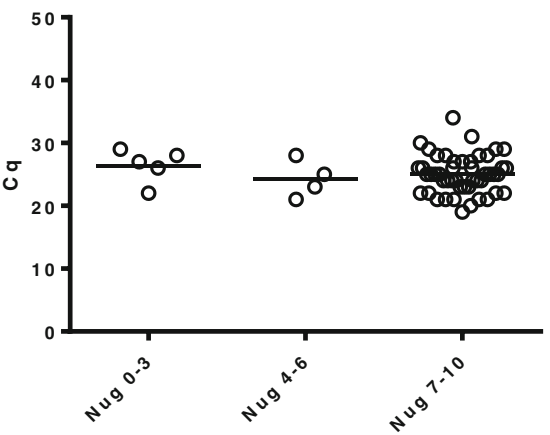

Fig. 1 Relation between $\mathrm{Cq}$ values and Nugent scores. Each panel represents the $\mathrm{Cq}$ values obtained in the multiplex qPCR for each of the five bacterial species tested. Circles represent Nugent score and $\mathrm{Cq}$ values of a single patient grouped per patient category. Nugent scores $0-3$

\section{Discussion}

The present study describes the development and clinical validation of a multiplex qPCR for the molecular diagnosis of BV. The vaginal microbiota is composed of numerous bacterial species, and recently researchers sought for correlations between the presence or absence of these species and the clinical condition of BV [5-10, 12, 13, 15]. Building on these previous reports, we selected three BV-marker organisms to include in our multiplex PCR. In addition, two Lactobacillus spp. were incorporated in the qPCR as targets. Lactobacillus spp. are believed to play an important role in maintaining a healthy vaginal environment [16], and where L. crispatus has been found mainly in healthy vaginal microbiota, $L$. iners is prevalent in both healthy and disturbed vaginal microbiota. As the vaginal microbial environment is dynamic, and transient phases between healthy microbiota and BV occur, we sought to incorporate these two Lactobacillus spp. in a quantitative relation to each other, in order to enable classification of transient phases in vaginal specimens. Besides, the relative quantitation of Gram positive rods, morphology consistent with Lactobacillus spp., constitutes a significant component in the frequently performed Gram-stain for diagnosing BV (indicating normal), 4-6 (intermediate) and 7-10 (indicative of bacterial vaginosis) are presented on the $\mathrm{X}$-axis, and $\mathrm{Cq}$ scores on the $\mathrm{Y}$-axis. This figure represents the results of all 151 patient samples analysed in this study (see also Table 2)

and therefore it seemed reasonable to incorporate Lactobacillus species in a molecular test for BV.

We applied the multiplex qPCR (BV-PCR) in a prospective study among females with complaints of abnormal vaginal discharge. In contrast to previous studies for molecular diagnostics of BV [5, 8, 15], participants were not recruited from an STD clinic or among pregnant women. Indeed, having an STI, engagement in same sex behaviour or the amenorrheic state in pregnancy can influence vaginal microbiota $[17,18]$ and therefore clinical validation of a diagnostic test in samples from study participants recruited through general practitioners and gynaecologists like in our study seems more appropriate. The additional information obtained from the participants revealed no significant differences between the groups with NS 0-3 and NS 7-10 with regard to either age, contraceptive use, previous reported abnormal vaginal discharge, or other putative causes of vaginal discharge being present.

In the clinical validation of our assay, we observed that the presence of the combination of BV-marker organisms results in a better association with BV than the individual scores of either of these bacteria. Adding the Lactobacillus-index, which on its own was not predictive for BV, allowed classification of those samples in which only one BV-marker organism was detected. Compared to the reference method (Nugent 
Score on vaginal specimen), our BV-PCR showed a high sensitivity (92\%) and specificity (96\%) for diagnosis of BV, confirming the potential diagnostic value of molecular diagnosis of BV. Also in this study we found initially $28 \%$ of the vaginal smears discrepant in Nugent score as classified by two experienced technicians, whereas PCR results were highly reproducible and technician independent (data not shown). Furthermore, in contrast to the Nugent scoring system, in which abundant numbers of Lactobacilli (Gram positive rods) are scored as healthy vaginal microbiota, discrimination and quantification of the two Lactobacillus species in the qPCR generates information on a shift of Lactobacillus populations in the vagina. Recently, both in studies detecting Lactobacillus by qPCRs $[5,9,18]$ or by broad range $16 \mathrm{~s}$ rRNA PCRs [12, 19-21], L. iners was the most prevalent species in the vagina, but in contrast to L. crispatus it is common and abundant in vaginal microbiotas with high concentrations of BV-marker organisms [11, 12]. Also in our study we found significantly higher loads of $L$. iners (i.e. L-index $<1$ ) in women with BV, although in nearly half of the women without BV a higher load of $L$. iners compared to $L$. crispatus (i.e. L-index $<1$ ) was detected too. Whether this condition reflects a transient phase from predominance of healthy to disturbed vaginal microbiota requires follow-up studies within the individual female.

Although in previous clinical studies high loads of $G$. vaginalis and $A$. vaginae have been shown to correlate with a diagnosis of BV $[8,15]$, recently substantial variability in G. vaginalis loads with the menstrual cycle has been demonstrated [22]. We defined BV-PCR positivity relying on the composition of vaginal microbiota rather than the individual loads of the targeted BV-marker organisms. Additionally we used the load of different Lactobacillus species in indicating a shift in vaginal microbiota. Thus we reached an excellent sensitivity and specificity for the BV-PCR assay. As with Nugent based scoring also the BV-PCR test resulted in inconclusive results for a small number of patients (respectively, $9 \%$ and $7 \%$ ). From preliminary studies (data not shown), apart from G. vaginalis and $A$. vaginae, Megasphaera phylotype 1 was the most promising in molecular based diagnosis of bacterial vaginosis. It is possible that by adding other relevant species to a multiplex PCR the molecular diagnosis of BV can be even more refined compared to our BV-PCR assay.

In conclusion, compared to the reference Nugent score in females suffering from abnormal vaginal discharge, the described multiplex qPCR was found to be a reliable tool for diagnosis of BV. The main advantage of the BV-PCR is that it can be performed in a reproducible and standardized fashion that does not depend on subjective interpretation of the examiner as is the case with the frequently used scorings such as classical wet mount microscopy or Nugent score [23]. In addition this qPCR based method can easily be incorporated in a fully automated PCR workflow as the Qiagen QIAsymphony and the Roche FLOW, thus expanding the existing portfolio of high through-put routine diagnostic tests of a modern clinical microbiological laboratory. Further development of this assay might ultimately allow to translate it into a cheap and reliable point-of-care test. Since BV is a common, often transient but recurrent clinical condition, this diagnostic BV-PCR can be applied in follow-up diagnostics within the same female. As such it is suitable to elucidate the role of the different targeted species in the pathogenesis of bacterial vaginosis.

Acknowledgment The authors thank K. Gerritsen-Stevens for technical assistance and Dr. S. Svraka-Latifovic for critically reviewing the manuscript.

Funding This study was carried out without a specific grant from any funding agency in the public, commercial, or not-for-profit sectors.

Transparency declarations The authors have no competing interests.

Ethical approval of this study The use of human subjects in this study has been approved by the local ethical board of the investigating institute (Tergooi Hospital, Hilversum, the Netherlands). As part of this approval written and oral informed consent was obtained for the use of the samples and information obtained from the participants of this study.

Open Access This article is distributed under the terms of the Creative Commons Attribution 4.0 International License (http:// creativecommons.org/licenses/by/4.0/), which permits unrestricted use, distribution, and reproduction in any medium, provided you give appropriate credit to the original author(s) and the source, provide a link to the Creative Commons license, and indicate if changes were made.

\section{References}

1. Dekker JH (2005) The Dutch Health Council report on screening for Chlamydia: too reserved. Ned Tijdschr Geneeskd 149(16):850 852

2. Myer L, Denny L, Telerant R, Souza M, Wright TC Jr, Kuhn L (2005) Bacterial vaginosis and susceptibility to HIV infection in South African women: a nested case-control study. J Infect Dis 192(8):1372-1380

3. Amsel R, Totten PA, Spiegel CA, Chen KC, Eschenbach D, Holmes KK (1983) Nonspecific vaginitis. Diagnostic criteria and microbial and epidemiologic associations. Am J Med 74(1):14-22

4. Nugent RP, Krohn MA, Hillier SL (1991) Reliability of diagnosing bacterial vaginosis is improved by a standardized method of gram stain interpretation. J Clin Microbiol 29(2):297-301

5. Fredricks DN, Fiedler TL, Thomas KK, Oakley BB, Marrazzo JM (2007) Targeted PCR for detection of vaginal bacteria associated with bacterial vaginosis. J Clin Microbiol 45(10):3270-3276

6. Fredricks DN, Fiedler TL, Marrazzo JM (2005) Molecular identification of bacteria associated with bacterial vaginosis. N Engl J Med 353(18):1899-1911

7. Menard JP, Fenollar F, Henry M, Bretelle F, Raoult D (2008) Molecular quantification of Gardnerella vaginalis and Atopobium vaginae loads to predict bacterial vaginosis. Clin Infect Dis 47(1): 33-43

8. Menard JP, Mazouni C, Fenollar F, Raoult D, Boubli L, Bretelle F (2010) Diagnostic accuracy of quantitative real-time PCR assay 
versus clinical and Gram stain identification of bacterial vaginosis. Eur J Clin Microbiol Infect Dis 29(12):1547-1552

9. Zozaya-Hinchliffe M, Lillis R, Martin DH, Ferris MJ (2010) Quantitative PCR assessments of bacterial species in women with and without bacterial vaginosis. J Clin Microbiol 48(5):1812-1819

10. Cartwright CP, Lembke BD, Ramachandran K, Body BA, Nye MB, Rivers CA, Schwebke JR (2012) Development and validation of a semiquantitative, multitarget PCR assay for diagnosis of bacterial vaginosis. J Clin Microbiol 50(7):2321-2329

11. De Backer E, Verhelst R, Verstraelen H, Alqumber MA, Burton JP, Tagg JR, Temmerman M, Vaneechoutte M (2007) Quantitative determination by real-time PCR of four vaginal Lactobacillus species, Gardnerella vaginalis and Atopobium vaginae indicates an inverse relationship between L. gasseri and L. iners. BMC Microbiol 7:115

12. Srinivasan S, Hoffman NG, Morgan MT, Matsen FA, Fiedler TL, Hall RW, Ross FJ, McCoy CO, Bumgarner R, Marrazzo JM, Fredricks DN (2012) Bacterial communities in women with bacterial vaginosis: high resolution phylogenetic analyses reveal relationships of microbiota to clinical criteria. PLoS One 7(6), e37818

13. Dumonceaux TJ, Schellenberg J, Goleski V, Hill JE, Jaoko W, Kimani J, Money D, Ball TB, Plummer FA, Severini A (2009) Multiplex detection of bacteria associated with normal microbiota and with bacterial vaginosis in vaginal swabs by use of oligonucleotide-coupled fluorescent microspheres. J Clin Microbiol 47(12):4067-4077

14. Fredricks DN (2011) Molecular methods to describe the spectrum and dynamics of the vaginal microbiota. Anaerobe 17(4):191-195

15. Bradshaw CS, Tabrizi SN, Fairley CK, Morton AN, Rudland E, Garland SM (2006) The association of Atopobium vaginae and Gardnerella vaginalis with bacterial vaginosis and recurrence after oral metronidazole therapy. J Infect Dis 194(6):828-836
16. Antonio MA, Hawes SE, Hillier SL (1999) The identification of vaginal Lactobacillus species and the demographic and microbiologic characteristics of women colonized by these species. J Infect Dis 180(6):1950-1956

17. Marrazzo JM, Thomas KK, Fiedler TL, Ringwood K, Fredricks DN (2010) Risks for acquisition of bacterial vaginosis among women who report sex with women: a cohort study. PLoS One 5(6), e11139

18. Jakobsson T, Forsum U (2007) Lactobacillus iners: a marker of changes in the vaginal flora? J Clin Microbiol 45(9):3145

19. Oakley BB, Fiedler TL, Marrazzo JM, Fredricks DN (2008) Diversity of human vaginal bacterial communities and associations with clinically defined bacterial vaginosis. Appl Environ Microbiol 74(15):4898-4909

20. Spear GT, Sikaroodi M, Zariffard MR, Landay AL, French AL, Gillevet PM (2008) Comparison of the diversity of the vaginal microbiota in HIV-infected and HIV-uninfected women with or without bacterial vaginosis. J Infect Dis 198(8):1131-1140

21. Ravel J, Gajer P, Abdo Z, Schneider GM, Koenig SS, McCulle SL, Karlebach S, Gorle R, Russell J, Tacket CO, Brotman RM, Davis CC, Ault K, Peralta L, Forney LJ (2011) Vaginal microbiome of reproductive-age women. Proc Natl Acad Sci USA 108(Suppl 1): 4680-4687

22. Srinivasan S, Liu C, Mitchell CM, Fiedler TL, Thomas KK, Agnew KJ, Marrazzo JM, Fredricks DN (2010) Temporal variability of human vaginal bacteria and relationship with bacterial vaginosis. PLoS One 5(4), e10197

23. Forsum U, Jakobsson T, Larsson PG, Schmidt H, Beverly A, Bjornerem A, Carlsson B, Csango P, Donders G, Hay P, Ison C, Keane F, McDonald H, Moi H, Platz-Christensen JJ, Schwebke J (2002) An international study of the interobserver variation between interpretations of vaginal smear criteria of bacterial vaginosis. APMIS 110(11):811-818 